\title{
Ein hybrider Ansatz zur Unterstützung kollaborativer Designtechniken
}

\author{
Florian Geyer, Ulrike Pfeil, Anita Höchtl, Jochen Budzinski, Harald Reiterer \\ Arbeitsgruppe Mensch-Computer Interaktion, Universität Konstanz

\section{Zusammenfassung} \\ Dieser Artikel stellt einen hybriden Ansatz zur Unterstützung kollaborativer Designtechniken vor. Die \\ anhand der Designtechnik Affinity Diagramming beschriebenen Interaktionskonzepte werden aus einer \\ Literaturrecherche und einer empirischen Studie abgeleitet. Diese Analyse zeigt, dass die Nutzung von \\ Papierartefakten insbesondere in divergenten Phasen von Vorteil ist. Des Weiteren wird aufgezeigt, \\ welche Auswirkung häufige Wechsel zwischen Arbeitsflächen in konvergenten Phasen haben können. \\ Die auf Basis dieser Erkenntnisse entwickelten Interaktionskonzepte kombinieren Papierartefakte mit \\ interaktiven Displays zur Unterstützung kreativer Arbeitsabläufe.
}

\section{$1 \quad$ Einleitung}

Hybride Benutzerschnittstellen (Kirk et al. 2009) vereinen direkt-manipulative Interaktion auf Displays (z.B. Multi-touch) mit physischen Objekten (z.B. Tokens). Dieser Ansatz besitzt ein großes Potential für kollaborative Anwendungsdomänen, da er einer Gruppe von Benutzern einen flüssigen und unkomplizierten Zugang zu digitalen Informationen ermöglicht. Zugleich fördern begreifbare Benutzerschnittstellen (TUIs) gleichberechtigte und spielerische Explorationen und können daher auch einen positiven Einfluss auf die Motivation der Benutzer und die Sichtbarkeit von Aktionen haben (Hornecker 2002). Gerade für kreative Gruppenarbeiten wird daher besonders viel Potenzial gesehen, da in diesem Umfeld Technologie bisher eher hinderlich war. Desktopsysteme erzwingen zum Beispiel einen extremen Wechsel zwischen Gruppenaktivität und Einzelinteraktion, was zu einer Störung kreativer Arbeitsabläufe führt (Terry \& Mynatt 2002). Hybride interaktive Anwendungen setzen jedoch auch immer einen sinnvollen Übergang zwischen physischer und digitaler Interaktion voraus. Es ist eine große Herausforderung beide Welten sinnvoll in einen Arbeitsablauf zu integrieren und dabei auch die Stärken und Schwächen der jeweiligen Technologien zu berücksichtigen.

Der in diesem Artikel vorgestellte Ansatz zeigt beispielhaft auf, wie hybride Interaktionstechniken zur Unterstützung von Designaktivitäten eingesetzt werden können. Die Interakti- 
onskonzepte werden aus einer Literaturrecherche und der empirischen Beobachtung und Analyse einer kollaborativen Designtechnik abgeleitet und anhand einer prototypischen Umsetzung aufgezeigt (siehe Abb. 1). Im Folgenden werden zunächst verwandte Arbeiten vorgestellt. Anschließend werden die Ergebnisse der Literaturrecherche und der Analyse beschrieben. Daraufhin wird das resultierende Interaktionskonzept erläutert, bevor weitergehende Forschungsarbeiten diskutiert werden.
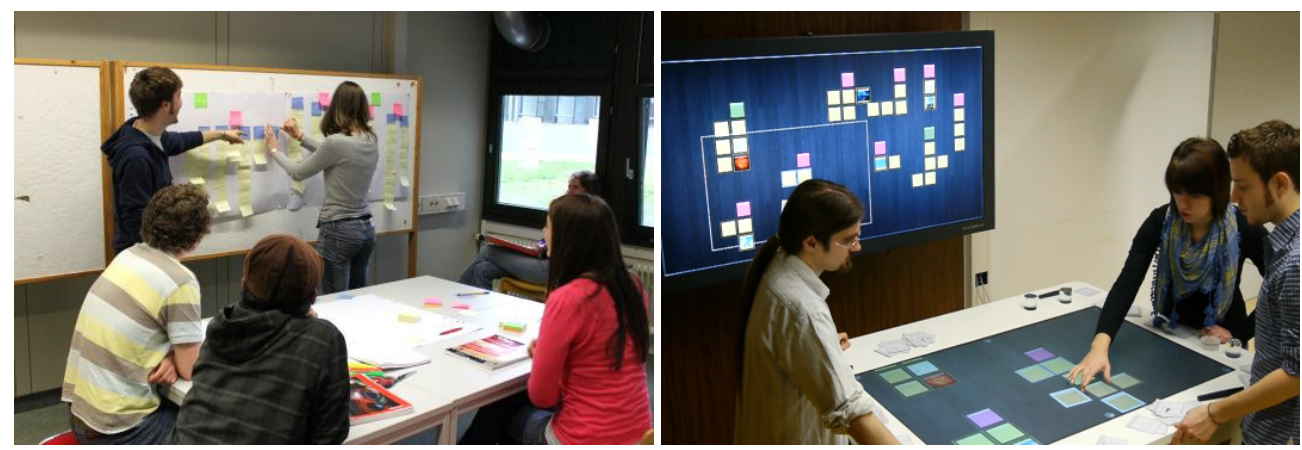

Abbildung 1: Das in diesem Artikel vorgestellte Interaktionskonzept (rechts) basiert auf einer Beobachtung und Analyse der kollaborativen Designtechnik Affinity Diagramming (links).

\section{Verwandte Arbeiten}

Ein richtungweisendes Konzept zur Unterstützung von Gruppenarbeiten an WebsiteStrukturen wurde mit The Designer's Outpost vorgestellt (Klemmer et al. 2001). Das System kombiniert Haftnotizen mit einem interaktiven Whiteboard. Durch eine über dem vertikalen Display angebrachte Kamera können die Notizen digitalisiert und daraufhin um virtuelle Verknüpfungen ergänzt werden. In ähnlicher Weise werden bei DigiPost (Jiang et al. 2007) Haftnotizen für die kollaborative Annotation von digitalen Karten auf einem horizontalen Display verwendet. Das System verwendet für die Interaktion mit der Software und für die Digitalisierung der Notizen die Digital Pen \& Paper $^{l}$ Technologie. Haller et al. (2006) zeigen mit dem Shared Design Space weitere Möglichkeiten zur Integration von Papier auf stiftbasierten digitalen Tischen auf. Eine optische Markererkennung ermöglicht hier eine direkte Kombination von digitalen Bildern und Papier zur Erstellung von Zeichnungen. Auf ähnliche Art werden in Pictionaire (Hartmann et al. 2010) digitale Bilder auf Papier projiziert um Annotationen zu ermöglichen. Neben papierbasierten Interaktionstechniken sind auch Konzepte für die Gestaltung der physischen Arbeitsumgebung relevant für diese Arbeit, da diese einen direkten Einfluss auf den Arbeitsablauf und die sozialen Aspekte von Gruppenarbeit haben. So wurde gezeigt, dass eine Kombination aus mehreren interaktiven Displays für kreative Gruppenarbeit von Vorteil sein kann. BrainStorm (Hilliges et al. 2007)

${ }^{1}$ www.anoto.com 
kombiniert einen stiftbasierten, interaktiven Tisch mit einer großflächigen Wandprojektion zur Unterstützung von Brainstorming-Prozessen. Digitale Notizzettel werden auf dem Tisch erstellt und können anschließend an der Wandprojektion mittels Stiftinteraktion verteilt und gruppiert werden. TeamStorm (Hailpern et al. 2007) kombiniert mobile Tablet PCs mit einem interaktiven Whiteboard für die kollaborative Erstellung und Diskussion von digitalen Skizzen.

Eine bisher weniger beachtete Fragestellung ist jedoch, wie hybride Benutzerschnittstellen in den Arbeitsablauf kollaborativer Designtechniken integriert werden können und welche Mehrwerte dadurch in Bezug auf typische Merkmale kreativer Prozesse entstehen können.

\section{Analyse}

Um eine Anwendung in einem realistischen Kontext zu erforschen, wurde eine Analyse durchgeführt, deren Ergebnisse im Folgenden beschrieben werden. Basierend auf einer Literaturrecherche werden einige wichtige Charakteristiken kollaborativer Designaktivitäten herausgearbeitet. In einem zweiten Schritt werden diese Ergebnisse für eine Beobachtung der Designtechnik Affinity Diagramming herangezogen. Durch eine quantitative und qualitative Analyse werden schließlich konkrete Probleme aufgezeigt, die als Basis für das Design eines Interaktionskonzepts dienen.

\subsection{Charakteristiken kollaborativer Designaktivitäten}

Kreative Aktivitäten werden oft als eine rapide, iterative Abfolge von Ideen-Generierung und Ideen-Bewertung beschrieben (Terry \& Mynatt 2002). Dabei wird die Phase der Generierung oft auch als divergent charakterisiert, da dort eine Vielzahl von unterschiedlichen Ideen entwickelt wird. Daraufhin folgt üblicherweise eine konvergente Phase, in der durch eine Bewertung aus übereinstimmenden oder ähnlichen Ideen eine Synthese erzielt werden sollte (Herring et al. 2009). Der schnelle Wechsel zwischen diesen zwei Aktivitäten wird von Schön (1983) als Reflection-in-Action bezeichnet und als kritisches Merkmal erfolgreicher kreativer Arbeitsweisen beurteilt. Im Kontext von kollaborativen Designaktivitäten wird die divergente Phase oft individuell und parallel durchgeführt, während die konvergente Phase ein gemeinsames Verständnis und Abstimmung unter Gruppenmitgliedern voraussetzt. Dabei haben soziale Faktoren, wie Bewertungsangst oder gegenseitige Blockierung, einen erheblichen Einfluss auf die Effektivität (Warr \& O`Neill 2005). Deshalb werden von professionellen Designern oft Methoden und Designtechniken bevorzugt, die durch einen strukturierten Ablauf eine Kontrolle über soziale Interaktionen ermöglichen (Herring et al. 2009; Beyer \& Holtzblatt 1998). Die physische Umgebung und die Art der Interaktion mit Artefakten spielen dabei eine wichtige Rolle, da diese als Werkzeuge zum Ideen-Austausch verwendet werden. So beschreibt eine ethnographische Studie von Vyas et al. (2009), welche Rolle physische Arbeitsflächen in Design Studios für die Kommunikation und Koordination spielen. Aus der Studie geht hervor, dass in der Praxis vertikale und horizontale Arbeitsflächen (sog. Live Surfaces) unterschiedliche Charakteristiken aufweisen, die einen direkten Einfluss 
auf die Qualität der Kollaboration haben können. In diesem Zusammenhang stellen Cook und Bailey (2005) durch eine umfangreiche Befragung von Designern fest, dass Papier, als flexibles und einfach zu handhabendes Artefakt in Gruppenaktivitäten eine hohe Akzeptanz genießt. Dies ist unter anderem durch die unkomplizierten Eigenschaften von Papier zu erklären, die den kreativen Fluss einer Gruppenaktivität genauso unterstützen wie die sozialen Interaktionen innerhalb der Gruppe. Zusammenfassend betonen wir folgende Charakteristiken: i) die Rolle sozialer Interaktion in divergenten und konvergenten Phasen, ii) der Einfluss der physischen Arbeitsumgebung auf die Designaktivität und iii) den Umgang mit Papierartefakten.

\subsection{Beobachtung der Designtechnik Affinity Diagramming}

Auf Basis einer Literaturrecherche alleine ist es nur schwer möglich konkrete Interaktionskonzepte abzuleiten. Es ist daher nötig ein tieferes Verständnis dafür zu erlangen, wie sich die beschriebenen Charakteristiken in der Praxis manifestieren und miteinander interagieren. Daher wurden die oben genannten Merkmale anhand einer typischen Designtechnik genauer untersucht. Als ein geeignetes Beispiel stellte sich Affinity Diagramming, beschrieben von Beyer und Holtzblatt (1998), heraus. Diese Methode ist eine weit verbreitete Designtechnik, die in ähnlicher oder abgewandelter Weise in Gruppen praktiziert wird. Beyer und Holtzblatt (1998) empfehlen für diese Technik explizit die Verwendung von Papierartefakten. Der grundsätzliche Arbeitsablauf besteht aus drei Phasen: In der ersten Phase werden von den Teilnehmern individuell Ideen oder Anregungen zu einem zuvor festgelegten Designproblem auf Haftnotizen geschrieben (divergente Phase). In der zweiten Phase werden diese Ideen rundenbasiert den anderen Teilnehmern präsentiert und auf eine gemeinsame Arbeitsfläche übertragen. Die dritte Phase dient dazu, die Haftnotizen auf Basis ihrer Ähnlichkeit (Affinität) so anzuordnen, dass ein gemeinsames Verständnis ermöglicht wird (konvergente Phase). Aufgrund dieses Ablaufs kann die Technik als typisch für eine Reihe von kollaborativen, papierbasierten Designtechniken betrachtet werden, die ein tieferes Verständnis eines Designproblems und dessen Lösung zum Ziel haben (Herring et al. 2009). Des Weiteren ist diese Technik durch ihre Rahmenbedingungen besonders geeignet, um die oben genannten Charakteristiken genauer zu untersuchen.

Es wurden drei Gruppen von Studenten (4-6 Studenten pro Gruppe, N=15) im Rahmen einer Vorlesung mit praktischer Übung zum Thema Interaktionsdesign beim Ausführen der Designtechnik beobachtet. Die Arbeitsumgebung (siehe Abb. 1, links) bestand, unter Berücksichtigung der Ergebnisse von Vyas (2009), aus einer horizontalen (Tisch) und einer vertikalen Arbeitsfläche (Pinnwand). Des Weiteren wurden verschiedenfarbige Haftnotizen und Stifte als Arbeitsmaterialen zur Verfügung gestellt. Nach einer Einführung in die Ziele und die Vorgehensweise der Designtechnik sowie das Designproblem (Gestenbasierte Interaktion mit einem Infotainmentsystem im Auto) führten die Studenten die Technik selbstständig aus. Die Gruppenaktivitäten wurden auf Video aufgezeichnet und zusätzlich von zwei Versuchsleitern mit Hilfe eines strukturierten Beobachtungsprotokolls erfasst. Eine Sitzung dauerte zwischen 1,5 und 2 Stunden. Basierend auf den Prinzipien von Interaction Analysis (Jordan \& Henderson 1995) und der qualitativen Inhaltsanalyse nach Mayring (2000), wurden für die in Kapitel 3.1 genannten Charakteristiken konkrete Kategorien entwickelt (Inter- 
aktion mit Papier, Aufmerksamkeit auf Arbeitsflächen und Soziale Interaktionen) und durch ein iteratives Verfahren in ein Kodierschema übertragen (siehe Abb. 2 und Abb. 3). Die Videoaufzeichnungen wurden aufgrund der identifizierten Phasen in Blöcke aufgeteilt, welches zusätzliche Information über die Unterschiede zwischen konvergenten und divergenten Aktivitäten liefert. Die jeweiligen Einzelaktivitäten der Teilnehmer wurden in Teilen von zwei Versuchsleitern kodiert. Eine Analyse der Interrater-Reliabilität ergab ein Cohens Kappa von 0.86, was als ausreichend angesehen wird. Die Frequenz der kodierten Aktivitäten gibt Aufschluss über die jeweilige Wichtigkeit in Bezug auf die drei Phasen der Designtechnik. Nach der Kodierung wurde die Videoaufzeichnung nach besonders auffälligen Kategorien gefiltert, um die betreffenden Videoausschnitte für eine qualitative Inhaltsanalyse heranzuziehen. Diese zweite Analyse ermöglichte es, Erklärungen für das Verhalten der Teilnehmer zu identifizieren (z.B. Warum halten die Teilnehmer Haftnotizen in der Hand?). Im Folgenden werden die wichtigsten Ergebnisse der Analyse innerhalb der Kategorien Interaktion mit Papier und Arbeitsflächen und Aufmerksamkeit zusammengefasst. Auf die Beschreibung der Ergebnisse aus der Kategorie Soziale Interaktionen muss aus Platzgründen verzichtet werden.

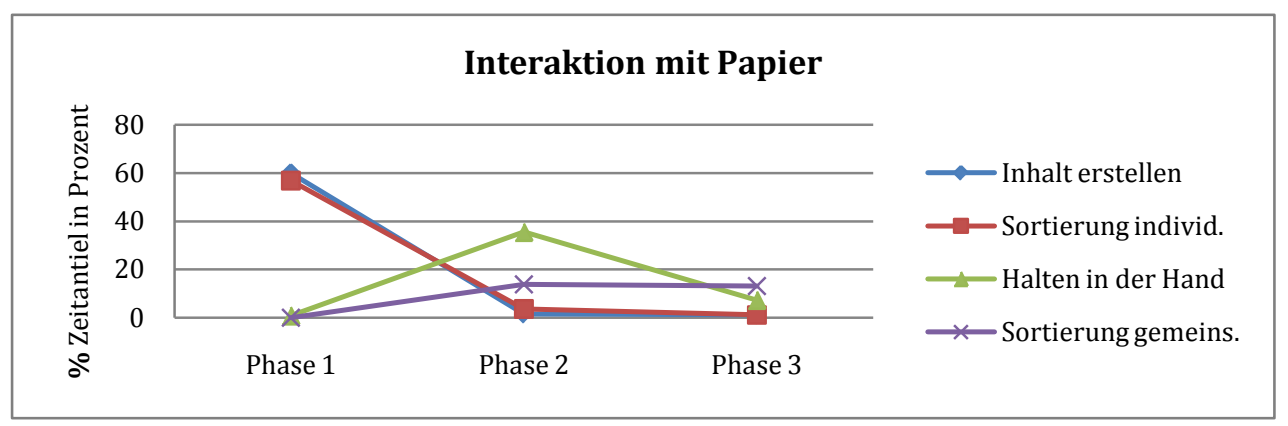

Abbildung 2: Frequenz und Art der Interaktionen mit Papier, verteilt auf die drei Kernphasen der Designtechnik.

Die Kategorien innerhalb des Aspektes Interaktion mit Papier waren: Die Erstellung von Inhalten, die Sortierung von Inhalten in einem persönlichen Arbeitsbereich, das Halten von Notizen in der Hand und die räumliche Anordnung von Papier an der Pinnwand. Abb. 2 zeigt die Frequenz dieser Aktivitäten, verteilt auf die drei Phasen der Designtechnik. Der Wert beschreibt den Anteil der Zeit in Prozent, den diese Aktivität für jeden Teilnehmer im Durchschnitt in der jeweiligen Phase eingenommen hat. So verbrachte ein Teilnehmer während der ersten Phase beispielsweise etwa 60 Prozent seiner Zeit für die Erstellung von Inhalten. Da die Kodierung der Aktivitäten nicht exklusiv durchgeführt wurde, überlappen sich diese Werte teilweise. Es konnte so jedoch festgestellt werden, dass die Erstellung von Inhalten untrennbar mit einer Sortierung im persönlichen Arbeitsbereich einhergeht. Eine inhaltliche Analyse dieser Aktivität ergab, dass die Teilnehmer die Sortierung in einem persönlichen Arbeitsbereich nutzen, um sich auf die Phase der Präsentation vorzubereiten oder um nach Inspiration zu suchen. Es konnte zudem beobachtet werden, dass diese Vorsortierung zu Problemen in der zweiten Phase führte, in der die Haftnotizen auf die gemeinsame Arbeitsfläche übertragen werden mussten. Hände, Teile der Arme und andere Objekte (z.B. Notiz- 
blöcke oder Bücher) wurden dazu verwendet, eine Reihe von Haftnotizen zwischen den Arbeitsbereichen zu transportieren. Diese Problematik führte dazu, dass die Teilnehmer in dieser Phase eine erhöhte Aufmerksamkeit auf Arbeitsflächen und Artefakte legten, und dadurch die Konzentration auf andere Gruppenmitglieder während der Präsentation gering blieb (siehe Abb. 3). Die Interaktion mit Haftnotizen in der dritten Phase war geprägt von Sortierungen in beiden Arbeitsbereichen und entsprechenden Transfer-Aktionen. Auch hier zeigten sich Probleme aufgrund des Abstandes zwischen den Arbeitsflächen. Es war zudem eine gegenseitige Blockierung der Teilnehmer durch eine eingeschränkte Sichtbarkeit und Erreichbarkeit der Haftnotizen auf der Pinnwand zu beobachten.

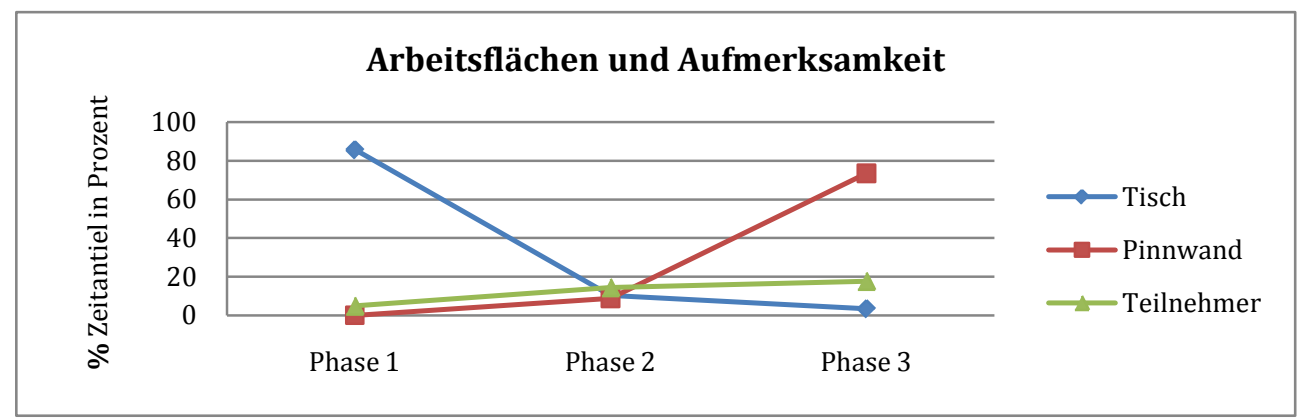

Abbildung 3: Verteilung der individuellen Aufmerksamkeit auf die drei Kernphasen der Designtechnik.

Innerhalb des Aspektes Arbeitsflächen und Aufmerksamkeit wurde zwischen der Aufmerksamkeit der Teilnehmer auf die Arbeitsflächen Tisch und Pinnwand sowie auf andere Personen unterschieden (siehe Abb. 3). Während in der ersten Phase meist still in den persönlichen Arbeitsbereichen gearbeitet wurde (86\%) und kaum soziale Interaktionen stattfanden, dominierte dagegen in der dritten Phase die Pinnwand als gemeinsamer Arbeitsbereich (74\%), begleitet mit erhöhter Aufmerksamkeit auf Gruppenmitglieder (17\%) während der Diskussion und Anordnung der Haftnotizen. Alle Gruppen verwendeten die Pinnwand als gemeinsamen Arbeitsbereich während der konvergenten Phase. Auffallend waren außerdem ein häufiger Wechsel der Aufmerksamkeit zwischen dem persönlichen und gemeinsamen Arbeitsbereich während der zweiten Phase und ein häufiger Wechsel zwischen Sortierung auf der Pinnwand und Diskussion unter den Teilnehmern in der dritten Phase. Es wurde zudem festgestellt, dass die Teilnehmer durch alle Phasen hinweg vom persönlichen Arbeitsbereich (Tisch) Gebrauch machten (siehe Abb. 2). Die Teilnehmer hatten teilweise Probleme der Gruppenarbeit zu folgen, wenn sie sich kurzzeitig in den persönlichen Bereich zurückgezogen hatten oder der gemeinsame Arbeitsbereich von anderen Teilnehmern verdeckt wurde. Während der Diskussion an der Pinnwand wurden zudem häufig Positionen gewechselt, um zwischen aktiver Sortierung der Haftnotizen, Betrachtung einer bestimmten Anordnung oder dem Überblick über die restlichen Haftnotizen zu wechseln. Dies führte jedoch zu einem erhöhtem Koordinationsaufwand in den Gruppen, der meist von einer Person übernommen wurde. 
Aus der Analyse ergaben sich zusammengefasst folgende Erkenntnisse: (i) Papier ist besonders in divergenten Phasen nützlich, da es Ideen-Generierung in einem semi-privaten Arbeitsbereich, sowie individuelle Reflektion effizient unterstützt (siehe Abb. 2); (ii) Der Transfer zwischen den Arbeitsbereichen und die Interaktion in konvergenten Phasen können jedoch mit visueller Blockierung verbunden sein; (iii) Die Aufmerksamkeit auf Arbeitsflächen und auf Personen ist vor allem in der konvergenten Phase durch einen häufigen Wechsel gekennzeichnet. Die physische Trennung der Arbeitsflächen kann dabei zu einem erhöhtem Koordinationsaufwand und einer unausgeglichenen Beteiligung der Teilnehmer führen.

\section{Ein hybrides Interaktionskonzept}

Im Folgenden wird ein hybrides Interaktionskonzept vorgestellt, das auf Basis der Erkenntnisse der Analyse in einem iterativen Designprozess entwickelt wurde. Es beschreibt konkrete Möglichkeiten den Gestaltungsfreiraum von hybriden Benutzerschnittstellen für die Unterstützung der Designtechnik Affinity Diagramming zu nutzen, um die gewohnten Abläufe physischer Praxis mit den Vorteilen digitaler Technologie zu vereinen. Der Ansatz umfasst drei grundlegende Konzepte:

Einsatz von Papier in divergenten Phasen. Unsere Ergebnisse zeigen, dass die Handhabung von Papier besonders in der ersten Phase von Vorteil ist. Zur Unterstützung individueller Arbeitsbereiche während divergenter Phasen und zur Erleichterung des Transfers in einen gemeinsamen Arbeitsbereich könnte daher Digital Pen \& Paper verwendet werden. Es bewahrt die gewohnte Möglichkeit der Erstellung und Vorsortierung in einem semi-privaten Arbeitsbereich. Durch eine direkte Digitalisierung der Handschrift oder von Handzeichnungen ist es zudem möglich, digitale Kopien ohne physische Einschränkungen in den gemeinsamen Arbeitsbereich zu übertragen. Dies kann beispielsweise durch ein Auflegen der Papierartefakte auf ein interaktives Display realisiert werden (siehe Abb. 4, links). Das Papier verbleibt anschließend für individuelle Reflektion und Manipulation im persönlichen Arbeitsbereich. Da die Stifte getrennt voneinander identifiziert werden können, ist es zusätzlich möglich, die Herkunft der digitalen Notiz nachzuverfolgen und eine Suche nach dem Autor der Notiz über ein personalisiertes Symbol anzubieten. Dies kann insbesondere in der konvergenten Phase für das gegenseitige Verständnis und zur Förderung der individuellen Beteiligung genutzt werden. 

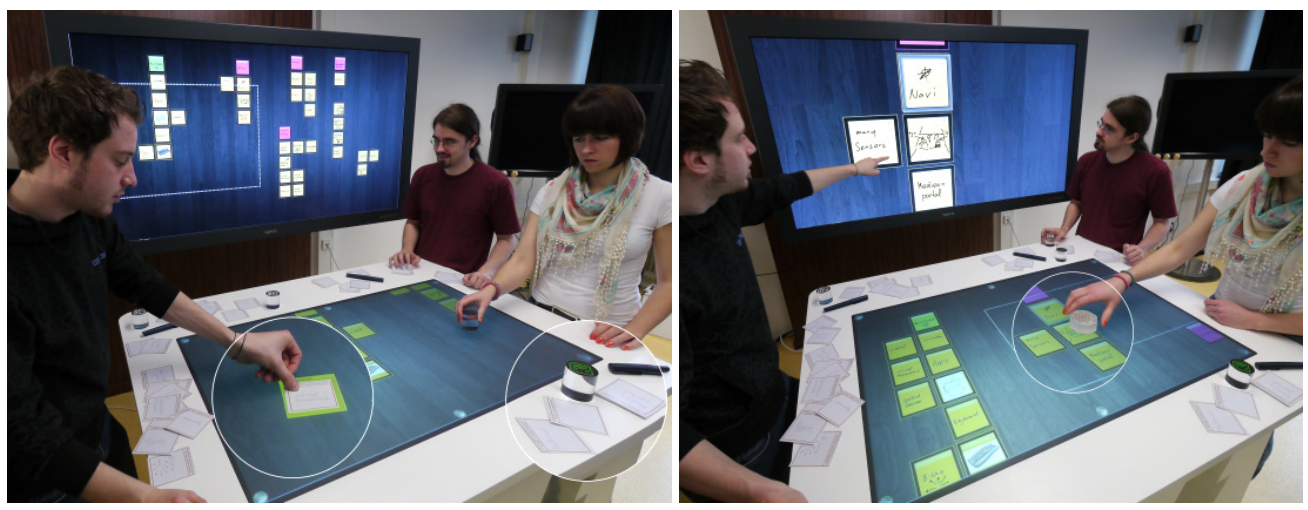

Abbildung 4: Persönliche Arbeitsbereiche am Tischrand ermöglichen individuelle Arbeit in divergenten Phasen; der Tisch bietet einen gemeinsamen Arbeitsbereich für konvergente Phasen; ein gekoppeltes, vertikales Display ermöglicht einen schnellen Wechsel zwischen Übersicht und Detail (links) sowie Aktion und Reflektion (rechts).

Vereinigung von horizontalen und vertikalen Arbeitsflächen. Wie unsere Beobachtung zeigte, können durch häufige Wechsel zwischen physisch getrennten Arbeitsbereichen gegenseitige Blockierung und Koordinationsprobleme entstehen. Durch einen gekoppelten Arbeitsbereich zwischen interaktiven Displays kann jedoch ein schnellerer Wechsel zwischen Aktion und Reflektion sowie Übersicht und Detail erreicht werden. Diese Vereinigung von Arbeitsflächen kann beispielweise durch die Kombination eines Multi-touch Tisches mit einem großen, hochauflösenden Wanddisplay (siehe Abb. 4, links) erfolgen. Der Tisch zeigt dabei nur einen verschiebbaren Ausschnitt des gesamten Arbeitsbereiches, der auf dem Wanddisplay vollständig dargestellt wird. Auf diese Weise kann, durch eine explizite Trennung der Arbeitsbereiche für Aktion und Reflektion, visuelle Blockierung reduziert werden. Der gemeinsame Arbeitsbereich bleibt so stets für alle Teilnehmer sichtbar. Die Verknüpfung der Arbeitsflächen muss jedoch durch entsprechende Visualisierungen deutlich gemacht werden um den kognitiven Mehraufwand dieser duplizierten Ansicht zu kompensieren. Dies kann durch eine Hervorhebung von fokussierten Artefakten (Highlighting) und durch eine Visualisierung des Fokusbereichs auf dem Kontextdisplay erreicht werden. Während Diskussionsphasen könnten zudem interaktive Detailansichten integriert werden, die beispielsweise durch Auflegen eines physischen Tokens auf den Tisch aufgerufen werden (siehe Abb. 4, rechts). Die Vergrößerung eines Ausschnittes des Arbeitsbereiches kann so als Koordinationswerkzeug genutzt werden.

Fokussierung der Interaktion in konvergenten Phasen. Wie unsere Studie zeigte, führt die physische Trennung der Arbeitsflächen besonders in der dritten Phase zu einer unausgeglichenen Beteiligung der Teilnehmer. Daher kann während konvergenter Phasen eine von allen Teilnehmern gut erreichbare Arbeitsfläche zur Förderung gleichberechtigter Beteiligung beitragen. So können Interaktionen, die eine gemeinsame Diskussion voraussetzen, durch eine räumliche Beschränkung der Arbeitsfläche und durch die Verwendung physischer Werkzeuge von den Teilnehmern besser nachverfolgt werden. Zum Beispiel kann ein Multitouch Tisch zur Fokussierung der Interaktion verwendet werden (siehe Abb. 4). Dies erlaubt eine realitätsnahe Anordnung der digitalen Kopien durch Multi-touch Interaktion. So können 
direkt-manipulative Interaktionstechniken und Gesten zur Manipulation (z.B. Farbänderung der Notizen) und Gruppierung eingesetzt werden. Physische Token (siehe Abb. 4, links) können zudem verwendet werden um typische Aufgaben wie das Löschen, Einsammeln und Weitergeben von Notizen aktiv zu unterstützen. Die hybride Digitalisierung dieser Aufgaben durch physische Token kann eine erhöhte Sichtbarkeit der Aktionen erreichen.

\section{Ausblick}

Dieser Artikel demonstriert am Beispiel einer kollaborativen Designtechnik die Herleitung eines hybriden Interaktionskonzepts zur Unterstützung kreativer Gruppenaktivitäten. Die vorgestellten Interaktionstechniken können in angepasster Weise für ähnliche Designmethoden verwendet werden (z.B. Sketching). Zudem adressieren die Kernkonzepte generische Phasen und Charakteristiken kreativer Gruppenarbeit. Der Artikel liefert jedoch keinen empirischen Nachweis über positive Auswirkungen der beschriebenen Interaktionstechniken. Es ist daher eine tiefergehende Evaluationsstudie nötig, um die Auswirkungen auf Effizienz, Effektivität und soziale Koordination in der Praxis zu untersuchen.

\section{Danksagung}

Wir danken der DFG für die Förderung dieser Arbeit (RE 1843/3-1). Dank gilt auch HansChristian Jetter und Michael Zöllner für die Entwicklung des Softwareframeworks ZOIL, das als technische Basis für die Umsetzung des Prototypen verwendet wurde.

\section{Literaturverzeichnis}

Beyer, H. \& Holtzblatt, K. (1998). Contextual design: defining customer-centered systems. San Francisco: Morgan Kaufmann.

Cook, D. J. \& Bailey, B. P. (2005). Designers' use of paper and the implications for informal tools. Proceedings of the 17th Australia Conference on Computer-Human Interaction: Citizens Online: Considerations for Today and the Future. ACM Press.

Hailpern, J., Hinterbichler, E., Leppert, C., Cook, D. \& Bailey, B. P. (2007). TEAM STORM: demonstrating an interaction model for working with multiple ideas during creative group work. Proceedings of the 6th ACM Conference on Creativity \& Cognition. ACM Press. 193-202.

Haller, M., Leithinger, D., Leitner, J., Seifried, T., Brandl, P., Zauner, J. \& Billinghurst, M. (2006). Shared design space: sketching ideas using digital pens and a large augmented tabletop setup. Advances in Artificial Reality and Tele-Existence. Lecture Notes in Computer Science. Springer. 185196.

Hartmann, B., Morris M.R., Benko H. \& Wilson, A.D. (2010). Pictionaire: supporting collaborative design work by integrating physical and digital artifacts. Proceedings of the 22nd ACM Conference on Computer Supported Cooperative Work. ACM Press. 421-424.

Herring, S. R., Jones, B. R. \& Bailey, B. P. (2009). Idea generation techniques among creative professionals. Proceedings of the 42nd Hawaii International Conference on System Sciences. IEEE Computer Society. 1-10. 
Hilliges, O., Terrenghi, L., Boring, S., Kim, D., Ritcher, H. \& Butz, A. (2007). Designing for collaborative creative problem solving. Proceedings of the 6th ACM Conference on Creativity \& Cognition. ACM Press. 137-146.

Hornecker, E. (2002). Understanding the benefits of graspable interfaces for cooperative use. Cooperative Systems Design: A Challenge of the Mobility Age. IOS Press. $71-87$.

Jiang, H., Yeh, R. B., Winograd, T. \& Shi, Y. (2007). DigiPost: writing on post-its with digital pen to support collaborative editing tasks on tabletop displays. Adjunct Proceedings of the 20th Annual ACM Symposium on User Interface Software and Technology. ACM Press.

Jordan, B. \& Henderson, A. (1995). Interaction analysis: foundations and practice. The Journal of the Learning Sciences, Vol. 4(1). 39-103.

Kirk, D., Sellen, A., Taylor, S., Villar, N. \& Izadi, S. (2009). Putting the physical into the digital: issues in designing hybrid interactive surfaces. Proceedings of the 23rd British HCI Group Annual Conference on People and Computers. British Computer Society. 35-44.

Klemmer, S.R., Newman, M.W., Farrell, R., Bilezikjian, M. \& Landay, J.A. (2001). The Designers' Outpost: A tangible interface for collaborative web site design. Proceedings of the 14th Annual ACM Symposium on User Interface Software and Technology. ACM Press. 1-10.

Mayring, P. (2000). Qualitative content analysis. Forum: Qualitative Social Research / Forum Sozialforschung FQS, Vol. 1(2). Art. 20.

Schön, D. A. (1983). The reflective practitioner: How professionals think in action. Basic Books.

Terry, M. \& Mynatt, E. D. (2002). Recognizing creative needs in user interface design. Proceedings of the 4th ACM Conference on Creativity \& Cognition. ACM Press. 38-44.

Vyas, D., Van Der Veer, G., Heylen, D. \& Nijholt, A. (2009). Space as a resource in creative design practices. Proceedings of the 12th IFIP TC13 Conference on Human-Computer Interaction: INTERACT 2009. Springer. 169-172.

Warr, A. \& O’Neill, E. (2005). Understanding design as a social creative process. Proceedings of the 5th ACM Conference on Creativity \& Cognition. ACM Press. 118-127. 